\title{
PERSONAL NAVIGATION ALGORITHMS BASED ON WIRELESS NETWORKS AND INERTIAL SENSORS
}

\author{
Zdeněk Kaňa - Zdeněk Bradáč — Petr Fiedler *
}

\begin{abstract}
The work aims at a development of positioning algorithm suitable for low-cost indoor or urban pedestrian navigation application. The sensor fusion was applied to increase the localization accuracy. Due to required low application cost only low grade inertial sensors and wireless network based ranging were taken into account. The wireless network was assumed to be preinstalled due to other required functionality (for example: building control) therefore only received signal strength (RSS) range measurement technique was considered. Wireless channel loss mapping method was proposed to overcome the natural uncertainties and restrictions in the RSS range measurements The available sensor and environment models are summarized first and the most appropriate ones are selected secondly. Their effective and novel application in the navigation task, and favorable fusion (Particle filtering) of all available information are the main objectives of this thesis.
\end{abstract}

K e y w ords: personal localization, indoor and urban navigation, wireless networks, inertial sensors, particle filter

\section{INTRODUCTION}

During last decades, the fast growth in wireless communication and electronics generally has enabled the development of microsensors that can interface with surroundings cordless. Since the price of these devices is decreasing, huge number of them concentrated in the wireless control network, Wireless Sensor Network (WSN), or a general wireless communication network can be deployed to cover outdoor, urban, or indoor areas.

Ubiquitous computing and growing usage of informational and electronic technologies in every-day life brings the need of location of things and persons. The terms like ubiquitous services, pervasive computing, networks convergence can be heard frequently nowadays. The fast development in the field of electronics has also enabled the practical realizations of ideas, which fits the smart city concept. The services are directed at the persons, inhabitants and are locally dependent. So the localization of the person is a crucial feature of such system.

The Global Navigation Satellite System (GNSS) or more specifically Global Positioning System (GPS) does not fit very well for these applications. The main disadvantages of GNSS are: vulnerability to disturbances, high energy consumption, relatively high price of quality receivers, and outdoor usage restriction. Although the last drawback can be partially overcome with the indoor GNSS repeaters [1], the solution accuracy and the installation costs are not on an applicable level yet. Many efforts have been concerned on development of Local Positioning System (LPS) during last years.

Seen from the perspective of wireless networks based positioning, the LPS layout consists of Reference Nodes $(\mathrm{RN})$ and Blindfolded Nodes (BN). RNs know their actual position, which can be set up by administrator at the installation of network or acquired by supreme positioning system (for example GPS in outdoor installations) and can be called beacons or anchor nodes. BNs calculate their location from measurements of ranges of RNs or other BNs and can be called moving nodes.

There must be solved sequent tasks to locate the RF device. Firstly the measurement/ communication technology must be chosen. Nowadays, these RF technologies are most commonly used: RF identification, ZigBee, Ultra Wideband (UWB) or Wi-Fi. Then there must exist RNs with prior knowledge of their location, independent on used LPS. The third step is to obtain the estimated ranges to neighboring RNs. The range estimation can be based on different measured physical variables: Received Signal Strength (RSS), Time of Arrival (TOA), Time Difference of Arrival (TDOA), or Angle of Arrival (AOA).

Next, Localization Algorithm (LA) needs to be involved to compute all the required coordinates of BNs. There have been proposed many LAs: Triangulation - usually used Least Mean Squares (LMS) approach when over-defined condition [2,3], Maximum Likelihood Estimation (MLE) [4,5], Signpost - Nearest neighbor method, Signal fingerprinting, etc.

Since the range measurement typically gives very rough data and also the deployment of various LAs do not return satisfactory outcomes, there has been paid a lot of attention to utilization of several localization improving techniques to precise the LA's estimation. Among them belong averaging (done in time, frequency or reciprocal channel level), or Bayesian techniques (Bayesian filters - BF: Kalman filter - KF, Grid-based method, particle filter - PF, multiple use of BFs, etc) [6].

The available computational resources (modern smartphones are equipped with powerful processors and sufficient amount of memory) enable the use of more complex, precise and reliable LAs. On the other hand, the demand to be independent on the wireless standard and low cost required is limiting the efforts to received signal strength (RSS) range measurement method, which is

* Department of Control and Instrumentation, Faculty of Electrical Engineering and Communication Brno University of Technology, Technická 3082/12, 61600 Brno, Czech Republic, kana.zdenek@gmail.com 
giving back only very perturbed data. IEEE 802.15.4a, an amendment to IEEE 802.15.4, which extends the physical layers with UWB and chirp spread spectrum techniques was introduced in 2006. Although these techniques enables deployment of TOA and TDOA based range estimation, the market with available devices is rather small, the price is higher as compared to other technologies and the communication is very unreliable.

The fusion with other sensors readings is a logical approach how to increase the positioning accuracy. Since the emphasis is put on a low cost solution the sensor set is quite narrow: inertial sensors (gyro, accelerometer) and magnetometer. Because the navigation algorithm must be applicable in indoor environment, where significant magnetic disturbances shall be assumed, the focus was put on inertial sensors only.

There exist two basic approaches for inertial sensors based pedestrian navigation. Firstly, a strap-down inertial navigation system (SINS) $[7,8]$ can be used. Since the sensors must be of low grade (cost and weight requirements) and the pedestrian motion is very complex to implement pedestrian navigation system based on SINS is a challenging task. The reason is that the aiding sources for such system (LPS or GNSS) won't be accurate enough to estimate inertial sensors errors with their given grade and pedestrian motion in play. There exists one very popular solution: the inertial sensors are attached to the pedestrian foot $[9,10]$. Then a zero velocity update [7] can be used during stance phase of stride to calibrate observable inertial sensor's biases.

The second approach is based on the pedestrian walking locomotion [11-13] and fits to the concept of this work better then SINS because the inertial sensors can be attached to the body of pedestrian or be worn in a pocket (some smart phones already includes inertial sensors and can be easily reused). The accelerometer measurements are used to detect the steps (the step length can be estimated, too) and together with gyro measurement constitutes a pedometer.

Since most of the measurement errors in the RSS based range estimation methods are due to the indoor environmental effects like is shadowing and multipath a technique to estimate the values and associated uncertainties of these effects can significantly increase the localization accuracy of the proposed solution.

The objective of this work is to develop a navigation algorithm, which will be implementable in the low-cost pedestrian navigation system and the assumption of preinstaled RF communication network can be taken.

\section{STATE OF THE ART}

\subsection{Wireless Network Based Positioning}

In this subsection, the first task - wireless network based positioning - is briefly described. There are two types of coordinates, estimated BN (sometimes called anchor nodes) and known RN (sometimes called beacon nodes).
The distance between nodes $i$ and $j$ (in 2-D) could be obtained with the use of triangulation

$$
d_{i, j}=\sqrt{\left(x_{i}-x_{j}\right)^{2}+\left(y_{i}-y_{j}\right)^{2}} .
$$

There are described three types of variations in the radio propagation channel $[15,16]$ :

- Small-scale variations (fast fading): These variations are caused by multipath character of the channel.

- Mid-scale variations (slow fading): They are mainly caused by shadowing and terrain contours and may exhibit great differences; the distance between nodes is equal.

- Large-scale variations (path loss): The increasing distance between nodes is dramatically changing the channel's structure and measured parameters statistic. RSS location technologies are based on this fact.

The choice of range measurement method is the crucial decision point; there can be found many comparisons in the literature [4]. The TOA method is based on measuring the time, which needs the signal to travel the distance between transmitter and receiver. In networks where the clocks are not synchronized is commonly used the TDOA technique, where the first device transmits a signal to the second, which replays on its receive. The time interval measured on the first device consists of twice TOA and the second device replay delay, which is either known or measured by the second device and then transmitted to the first device. TOA claims the use of accurate (expensive) clocks and the main sources of errors are the non-line of sight signals, which travels longer way then the main line of sight signal.

The AOA method is reporting the angle not the distance of neighbors and requires costly antenna arrays. The RSS method appears to be the cheapest one from all named, but also the least accurate one. The biggest advantage of RSS method is the fact that a Received Signal Strength Indicator (RSSI) is part of most communication standard.

The large-scale variations [17] in power path-loss over distance $d_{i, j}$ between nodes $i$ and $j$ is observing inverseexponential pattern, formulated in $\mathrm{dBm}$

$$
\tilde{P}_{i, j}(\mathrm{dBm})=P_{0}(\mathrm{dBm})-10 n_{p} \log \frac{\tilde{d}_{i, j}}{d_{0}}
$$

where $n_{p}$ is path-loss exponent, $P_{0}$ is received power at short reference distance $d_{0}$. There is typically used a lognormal (Gaussian if expressed in decibels) distribution for modeling the range measurement errors.

The LA computes from the measured ranges to RNs the position of $\mathrm{BN}$.

MLE maximizes of the probability of location solution based on the statistical character of the wireless propagation channel. By taking the negative logarithm and 
assuming all available measurements have the same variance, the 2-D position can be estimated as follows [4]

$$
\left[x_{1}, \ldots, x_{n} ; y_{1}, \ldots, y_{n}\right]=\underset{\left[x_{1}, \ldots, x_{n} ; y_{1}, \ldots, y_{n}\right]}{\arg \min }\left(\ln \frac{\tilde{d}_{i, j}^{2}}{d_{i, j}^{2}}\right)^{2} .
$$

Least mean square (LMS) algorithm minimizes the mean of differences squares and assumes identical measurement error characteristics. It is a methodology, which is appropriate also for overdetermined tasks, where there exist more measurements, than is needed for the task to be solvable. When the errors in measurements have the Gaussian distribution, the LMS solution tends to MLE solution.

When the assumption of identical measurement error distribution does not hold the truth, the LMS can be reformulated to WLMS.

\subsection{Bayesian Filtering In Positioning}

Since the "traditional" LAs (described previously) combined with RSS range measurements does not carry out sufficient outcomes, it is logical to proceed from snapsolutions (represented by the MLE, LMS and WLMS methods) to the filtered solutions. In filtered solutions, also the path history is used (for BFs only the actual and previous step), not only current measurements from the sensors. The probabilistic relationships between variables enable the utilization of conditional probability techniques, eg BFs. These conditional probabilities usually express both, the system dynamics (state evolution) and measurement models. These models can be and in this thesis will be used to describe the navigation system represented by the pedestrian moving along an area covered by the RF signal from multiple RNs.

The probabilistic approach used in BF means, that the state is not represented directly, by a numerical value, but by the belief $b e l(x(t))$, which assigns a probability to each possible solution.

Bayes filter consists of two essential steps:

(1) Prediction (Control update) - There is predicted the next state vector $\overline{b e l}(x(t))$ according to the previous state $\operatorname{bel}(x(t-1))$ and system dynamic model $p(x(t) \mid x(t-1))$.

$$
\overline{b e l}(x(t))=\int p(x(t) \mid x(t-1)) \operatorname{bel}(x(t-1)) \mathrm{d} x(t-1) .
$$

(2) Correction (Measurement update) - Sensor likelihood (perceptual) model $p(y(t) \mid x(t))$ is used for correction of the state predicted in the previous step.

$$
\operatorname{bel}(x(t))=\eta p(y(t) \mid x(t)) \overline{b e l}(x(t)) .
$$

The PF represents the belief by a set of $M$ weighted random state samples (6) drawn from this probability density function (7)

$$
\begin{gathered}
X(t)=x^{[1]}(t), x^{[2]}(t), \ldots, x^{[M]}(t), \\
\operatorname{bel}(x(t))=\sum_{i=1}^{M} w^{[i]} \delta\left(x-x^{[i]}\right) .
\end{gathered}
$$

The basic variant of PF consists of these steps [14]

$$
\begin{gathered}
\forall m \in[1, \ldots, M]: \text { sample } x^{[M]}(t) \\
\approx p\left(x(t) \mid u(t), x^{[m]}(t-1)\right), \\
w^{[m]}(t)=p\left(y(t)|| x^{[m]}(t)\right) \\
\overline{\mathrm{X}}(t)=\overline{\mathrm{X}}(t)+\left\langle x^{[m]}(t), w^{[m]}(t)\right\rangle
\end{gathered}
$$

$\forall m=[1, \ldots, M]:$ draw $i$ with probability $\propto w^{[i]}(t)$,

$$
\text { add } x^{[i]}(t) \text { to } \mathrm{X}(t) .
$$

Initially, there is generated a set of $M$ hypothetical states (8), each based on the control $u(t)$, particles from the last step $x(t-1)^{[m]}$ and system dynamic model $p(x(t) \mid u(t), x(t-1))$. To include the measurement $y(t)$ into the particle set, the so-called importance factor $w(t)^{[m]}$ is calculated in (9) for each particle. This step is called importance sampling. Equations (11) and (12) are representing the re-sampling procedure. This procedure draws from the set of predicted particles $\overline{\mathrm{X}}(t)$ a set of $M$ new particles $\mathrm{X}(t)$. Particles in $\overline{\mathrm{X}}(t)$ are distributed according to the $\overline{b e l}(x(t))$ and in the set $\mathrm{X}(t)$ according to the $\operatorname{bel}(x(t))=\eta p\left(y(t) \mid x^{[m]}(t)\right) \overline{b e l}(x(t))$.

\subsection{System Dynamics And Measurement Models}

System dynamics and measurements models are two important components of BF.

There can be seen a typical pattern of vertical and forward acceleration during the human walking on a flat floor on Fig. 1. The peak detection can be deployed for the step detection.

An approach for estimating the step length was proposed in [11]. The walking speed can be estimated according to the difference between maximum and minimum for vertical and/or forward acceleration; the relationship can be easily linearized.

Inertial sensors comprise accelerometer and gyroscope (gyro), which measure specific force and angular rate, respectively. Since 3-D navigation information is usually required, triads of these sensors are combined in an inertial measurement unit (IMU). The general sensor error model, which can be applied for both, gyro and accelerometer measurements, is shown in

$$
\tilde{\mathbf{X}}=(\mathbf{I}-\mathbf{M}) \mathbf{X}+\boldsymbol{b}+\mathbf{w}
$$

where $\mathbf{I}$ is identity matrix (3-by-3 for 3-dimensional coordinate system), $\mathbf{x}$ is the true value (true specific force or angular rates) and $\tilde{\boldsymbol{X}}$ is measured value (sensor output). 


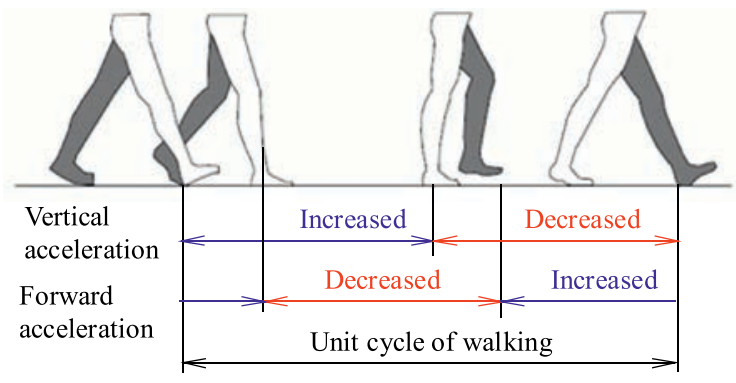

Fig. 1. Walking stage and acceleration pattern [11]

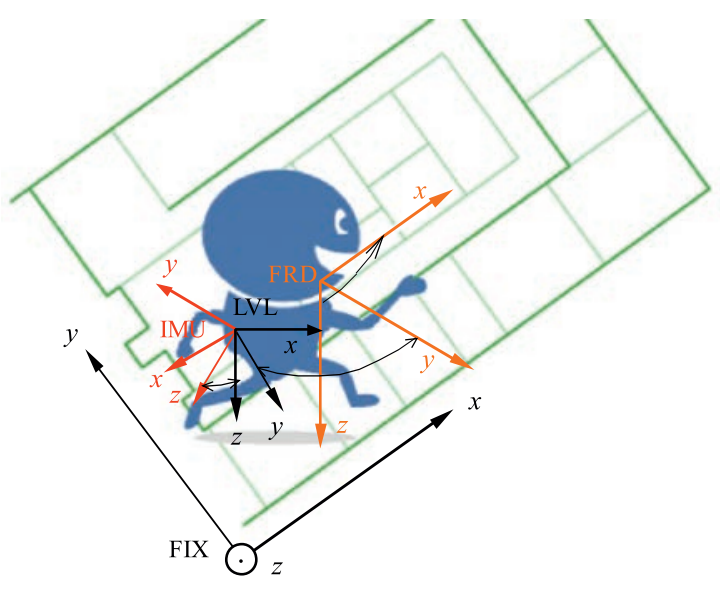

Fig. 2. Coordinate frames

$\boldsymbol{b}$ is the bias (typically the most remarkable error), $\mathbf{M}$ is the matrix of scale factors and misalignment errors, and $\boldsymbol{w}$ states for the measurement noise, typically assumed to be white (at least for frequency band constrained by the vehicle dynamics) and Gaussian.

The RF propagation channel model represents the measurement model in the application with framework of RNs, which are transmitting the RF signal and the BNs measures the ranges by the RSS method. As it has been said before, the range measurement errors are typically described with a log-normal (Gaussian if expressed in decibels) distribution.

\section{PROPOSED SOLUTION}

\subsection{Pedestrian Dead Reckoning Tightly Fused With RSS Based Range Measurements}

Four coordinate frames will be used and are depicted in Fig. 2.

The IMU (IMU) frame is fixed to the IMU and the accelerations and angular rates are measured in this frame. The leveled (LVL) frame has the same origin as IMU frame but is rotated in order the $\mathrm{z}$ axis was pointing down; this rotation is defined by two consecutive Euler rotations over two angles (roll and pitch). The forward-right-down (FRD) frame is a local leveled frame, too, this frame has its origin in the center of gravity of the pedestrian and the forward direction is aligned with the horizontal velocity vector projection. Although the leveled and forwardright-down frames are translated to each other, their relative rotation can be defined by a single vertical Euler rotation. The angle, which will be called IMU bearing, defines this rotation and is not easy to be estimated. Although some methodologies were proposed (ie [11]), the results of them are not satisfactory. In this work the IMU bearing will not be estimated and only vertical accelerations and angular rates will be further used. The fixed (FIX) frame is fixed to the environment, where the navigation should be resolved.

The system dynamics and measurement models are summarized in this subsection. The state space is to be described first

$$
\left[\begin{array}{llllllll}
r_{x}^{f i x} & r_{y}^{f i x} & \psi & b_{g y r, z}^{f r d} & \delta A_{\text {step }} & \delta B_{\text {step }} & \delta P_{0} & \delta n_{p}
\end{array}\right]^{\top}
$$

The state space consists of horizontal position $\left(r_{x}^{f i x}, r_{y}^{f i x}\right)$, heading $(\psi)$, vertical gyro bias $\left(b_{g y r, z}^{f r d}\right)$, errors of vertical acceleration and step length linearized relationship parameters (slope and intercept: $\delta A_{\text {step }}, \delta B_{\text {step }}$ ), error of received reference power coarse value $\left(\delta P_{0}\right)$ and error of path loss exponent coarse value $\left(\delta n_{p}\right)$.

The proposed discrete time system dynamics model is described in (15).

$$
\begin{aligned}
r_{x}^{f i x}(t) & =r_{x}^{f i x}(t-1)+\sin (\psi(t-1) \\
& \left.+\left(\tilde{\omega}_{z}^{f r d}-b_{g y r, z}^{f r d}(t-1)-w_{g y r, z}^{f r d}\right) \Delta t\right) S_{l e n}
\end{aligned}
$$

with $S_{l e n}=\left(\hat{A}_{\text {step }}+\delta A_{\text {step }}(t-1)\right)\left(\hat{a}_{z, \max }^{\text {frd }}\right.$

$$
\begin{aligned}
& \left.-\hat{a}_{z, \text { min }}^{f r d}\right) \Delta t_{\text {step }} \\
& +\left(\hat{B}_{\text {step }}+\delta B_{\text {step }}(t-1)\right) \Delta t_{\text {step }}+w_{\text {step }}, \\
& =r_{y}^{f i x}(t-1)+\cos (\psi(t-1) \\
& \left.+\left(\tilde{\omega}_{z}^{f r d}-b_{g y r, z}^{f r d}(t-1)-w_{g y r, z}^{f r d}\right) \Delta t\right) S_{l e n}
\end{aligned}
$$$$
r_{y}^{f i x}(t)=r_{y}^{f i x}(t-1)+\cos (\psi(t-1)
$$

with $S_{l e n}=\left(\hat{A}_{\text {step }}+\delta A_{\text {step }}(t-1)\right)\left(\hat{a}_{z, \max }^{\text {frd }}\right.$

$$
\begin{aligned}
& \left.-\hat{a}_{z, \min }^{\text {frd }}\right) \Delta t_{\text {step }} \\
& +\left(\hat{B}_{\text {step }}+\delta B_{\text {step }}(t-1)\right) \Delta t_{\text {step }}+w_{\text {step }},
\end{aligned}
$$$$
\psi(t)=\psi(t-1)+\left(\tilde{\omega}_{z}^{f r d}-b_{g y r, z}^{f r d}(t-1)-w_{g y r, z}^{f r d}\right) \Delta t,
$$

$$
\begin{aligned}
& b_{g y r, z}^{f r d}(t)=\exp \left(-\frac{\Delta t}{2 \tau_{g y r, z, G M}^{f r d}}\right) b_{g y r, z}^{f r d}(t-1)+w_{g y r, z, G M}^{\text {frd }}, \\
& \delta A_{\text {step }}(t)=\exp \left(-\frac{\Delta t}{2 \tau_{A_{\text {step }}}}\right) \delta A_{\text {step }}(t-1)+w_{A_{\text {step }} G M},
\end{aligned}
$$

$$
\begin{aligned}
\delta B_{\text {step }}(t) & =\exp \left(-\frac{\Delta t}{2 \tau_{B_{\text {step }}}}\right) \delta B_{\text {step }}(t-1)+w_{B_{\text {step }} G M} \\
\delta P_{0}(t) & =\exp \left(-\frac{\Delta t}{2 \tau_{P_{0}}}\right) \delta P_{0}(t-1)+w_{P_{0} G M} \\
\delta n_{p}(t) & =\exp \left(-\frac{\Delta t}{2 \tau_{n_{p}}}\right) \delta n_{p}(t-1)+w_{n_{p} G M}
\end{aligned}
$$

where $\tilde{\omega}_{z}^{\text {frd }}$ is measured horizontal angular rate with corresponding measurement noise $\left(w_{g y r, z}^{f r d}\right), \Delta t$ is system 


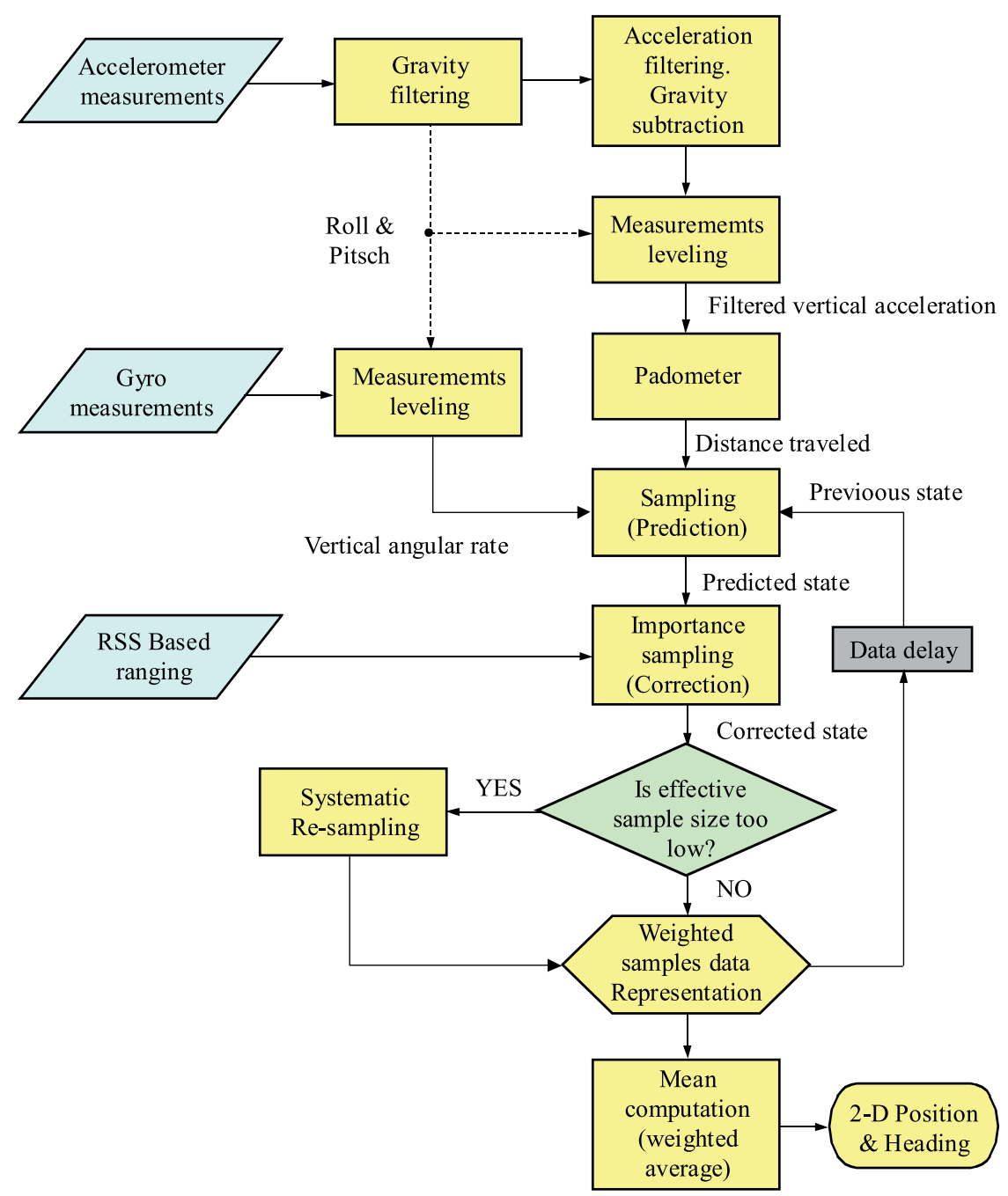

Fig. 3. Fusion filter flow chart

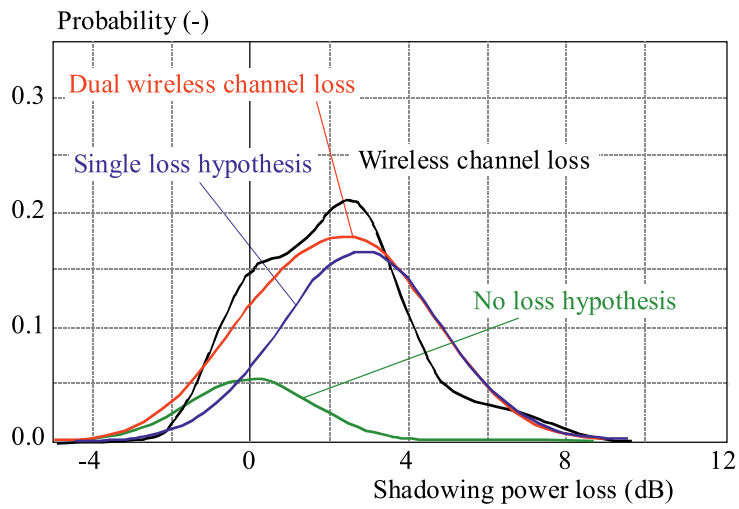

Fig. 4. Dual and full wireless channel loss mapping comparison

dynamics sampling time, $S_{l e n}$ is estimated step length with corresponding estimation noise $\left(w_{\text {step }}\right), \hat{A}_{\text {step }}$ and $\hat{B}_{\text {step }}$ are the slope and intercept of step length linearized relationship coarse estimates (to fit the wide range of human walk), $\hat{a}_{z, \max }^{f r d}$ and $\hat{a}_{z, \min }^{f r d}$ are the low-pass filtered vertical acceleration maximum and minimum used to estimate the step length. Vertical gyro bias, errors of ver- tical acceleration and step length linearized relationship parameters (slope and intercept), error of received reference power coarse value, and error of path loss exponent coarse value dynamics are modeled by first Gauss-Markov process with corresponding time constants $\left(\tau_{\ldots}\right)$ and driving noises $\left(w_{\ldots}\right)$.

The model is non-linear, but all the noises are modeled as white and Gaussian so the sampling (representing PF prediction) from the proposed system dynamic model is straightforward.

The number of available RSS ranging measurements to the $\mathrm{RN}$ will define the dimension of measurement vector. The measurement model is similar for the whole measurement vector

$$
\begin{aligned}
& \tilde{P}_{i}= \\
& \begin{aligned}
\hat{P}_{0}+\delta & P_{0}(t)-\hat{L}_{i}-5\left(\hat{n}_{p}+\delta n_{p}(t)\right) \log \left(\left(r_{x, R N, i}^{f i x}-r_{x}^{f i x}(t)\right)^{2}\right. \\
& \left.\quad+\left(r_{y, R N, i}^{f i x}-r_{y}^{f i x}(t)\right)^{2}+\Delta z^{2}\right)-w_{L_{i}}+w_{R S S}
\end{aligned}
\end{aligned}
$$

where $r_{x, R N, i}^{f i x}$ and $r_{y, R N, i}^{f i x}$ are the coordinates of RN $i$, $\Delta z$ is a constant to compensate the difference in the 
RN and $\mathrm{BN}$ height, $\hat{P}_{0}$ is the coarse value of reference receive power, $\hat{n}_{p}$ is path loss exponent coarse value, and $w_{R S S}$ is receiver noise. Finally, $\hat{L}_{i}$ is shadowing power loss with corresponding noise $\left.\left(w_{L_{i}}\right)\right)$, both variables will be discussed next.

The PF was selected to be used in proposed navigation algorithm. Since there exist many types of PF selected implementation is described. Sampling importance resampling particle filter with systematic re-sampling triggered by the estimated effective sample size threshold is to be used as part of the proposed navigation algorithm.

The sampling uncertainty is propagated based on the state space noises as defined in the system dynamics model (15) not based on an artificial constants as proposed in [14]. The flow chart shown on Fig. 3 depicts the simplified algorithm of fusion filter.

The accelerometer measurements are filtered with lowpass filter with relatively long time constant to estimate the Gravity. Then the specific force is filtered once again (low-pass filter with relatively short time constant) to lower the noise and the Gravity is subtracted next. Since the low-pass filter is not able to differ Gravity from the accelerometer bias, the bias is also inherently suppressed via this subtraction.

The estimated Gravity is used to compute the roll \& pitch angles and both, accelerometer and gyro measurements are leveled (the coordinate frame is rotated to have the $\mathrm{x}$ and $\mathrm{y}$ axis in the horizontal plane). As stated in the previous paragraph, the Gravity estimation includes also the accelerometer bias and the roll \& pitch will be just coarse estimates, but will be sufficient for proposed algorithms.

The vertical acceleration is used in the pedometer to detect the step (which triggers the new filter epoch) and to estimate the step length. The vertical angular rate and the distance traveled comprises the control signals used in the filter prediction step, which is propagating the particle set (representing the belief estimates from previous step) due to the state space noises. According to the RSS based ranging and measurement model, the samples are weighted. Afterwords, the weighted particle set effective sample size is enumerated and if it is lower than a predefined threshold the systematic re-sampling algorithm is applied on this particle set. In the end, the mean of estimated values are computed using the weighted average of the particle set.

\subsection{Dual Wireless Channel Loss Mapping}

There was propose (full/exhaustive) wireless channel loss mapping in [19] but since it is a complex algorithm, the number of variables (representing the hypothesis probabilities) can increase up to hundreds in the real applications and the solution of resulting system of equations can be very computationally demanding a simplified/ approximative method is proposed next.

Firstly, list of all relevant RN to RN hypotheses for every cell is constructed.
Secondly, when the RSS ranging is to be used, based on the predicted pedestrian $(\mathrm{BN})$ and $\mathrm{RN} i$ positions, a set $Q_{i}([x, y])$ of cells that intersects this measurement is constructed. This set of cells is used to build a set of all relevant $\mathrm{RN}$ to $\mathrm{RN}$ hypothesis $H_{i}(i, j)$ and the occurrence $\left(M_{c}\right)$ of each $\mathrm{RN}$ to $\mathrm{RN}$ hypothesis in these cells is enumerated. Only two (dual) hypotheses are constructed:

- There is a single loss with these characteristics

$$
\begin{aligned}
p_{L} & =1-\prod_{i, j \in H_{i}(i, j)}\left(1-M_{c} p_{R N i, j}\right), \\
P_{w, L} & =\frac{\sum_{i, j \in H(i, j)} M_{c} p_{R n i, j} P_{w R N i, j}}{\sum_{i, j \in H(i, j)} M_{c} p_{R n i, j}}, \\
\sigma_{P w, L}^{2} & =\left(1+\sum_{h=1}^{N_{H i}} \frac{1}{2 M_{c}}\right) \sigma_{R S S}^{2} .
\end{aligned}
$$

- There is no loss

$$
\begin{aligned}
P_{n L} & =1-p_{L}, \\
P_{w, n L} & =0, \\
\sigma_{P w, n L}^{2} & =\sigma_{R S S}^{2}
\end{aligned}
$$

where $p_{L}$ and $p_{n L}$ are the probabilities/ weights, $P_{w, L}$ and $P_{w, n L}$ are power losses, and $\sigma_{P w, L}$ and $\sigma_{P w, n L}$ are standard deviations of noise of single loss and no loss hypothesis, respectively. $\sigma_{R S S}$ is the standard deviation of receiver measurement noise.

Because probabilities are only approximative, there can occur a situation when the approximated probability of single loss hypothesis is $p_{L}>p_{L, t h r}$. Where $p_{L, t h r}$ is a maximum probability assigned to single loss hypothesis, which can be set up to 0.6. Then the single loss hypothesis is replaced by multi loss hypothesis

$$
\begin{aligned}
\hat{p}_{L} & =\frac{p_{L}}{\operatorname{ceil}\left(p_{L}-p_{L, t h r}\right)+1}, \\
\hat{P}_{w, n L} & =P_{w, n L}\left(\operatorname{ceil}\left(p_{L}-p_{L, t h r}\right)+1\right), \\
\hat{\sigma}_{P w, n L}^{2} & =\sigma_{P w, n L}^{2}\left(\operatorname{ceil}\left(p_{L}-p_{L, t h r}\right)+1\right)
\end{aligned}
$$

where "ceil" stands for round towards plus infinity.

There will be probably some cells in the navigation area, which are not intersecting with any RN to RN measurement; an average value is assigned to these cells. The allocation of power into one cell will be computed and linearly averaged over all RN to RN measurements

$$
L_{a v r}=\frac{\sum_{i, j \in H_{4}(i, j)} p_{R N i, j} P_{w R N i, j}}{N_{r}}
$$

where $H_{4}(i, j)$ is a set of $N_{r}$ elements, which contains all RN to RN measurements. If there exist $N_{\text {avr }}$ cells with no RN to RN hypothesis, both, the no-loss and single 


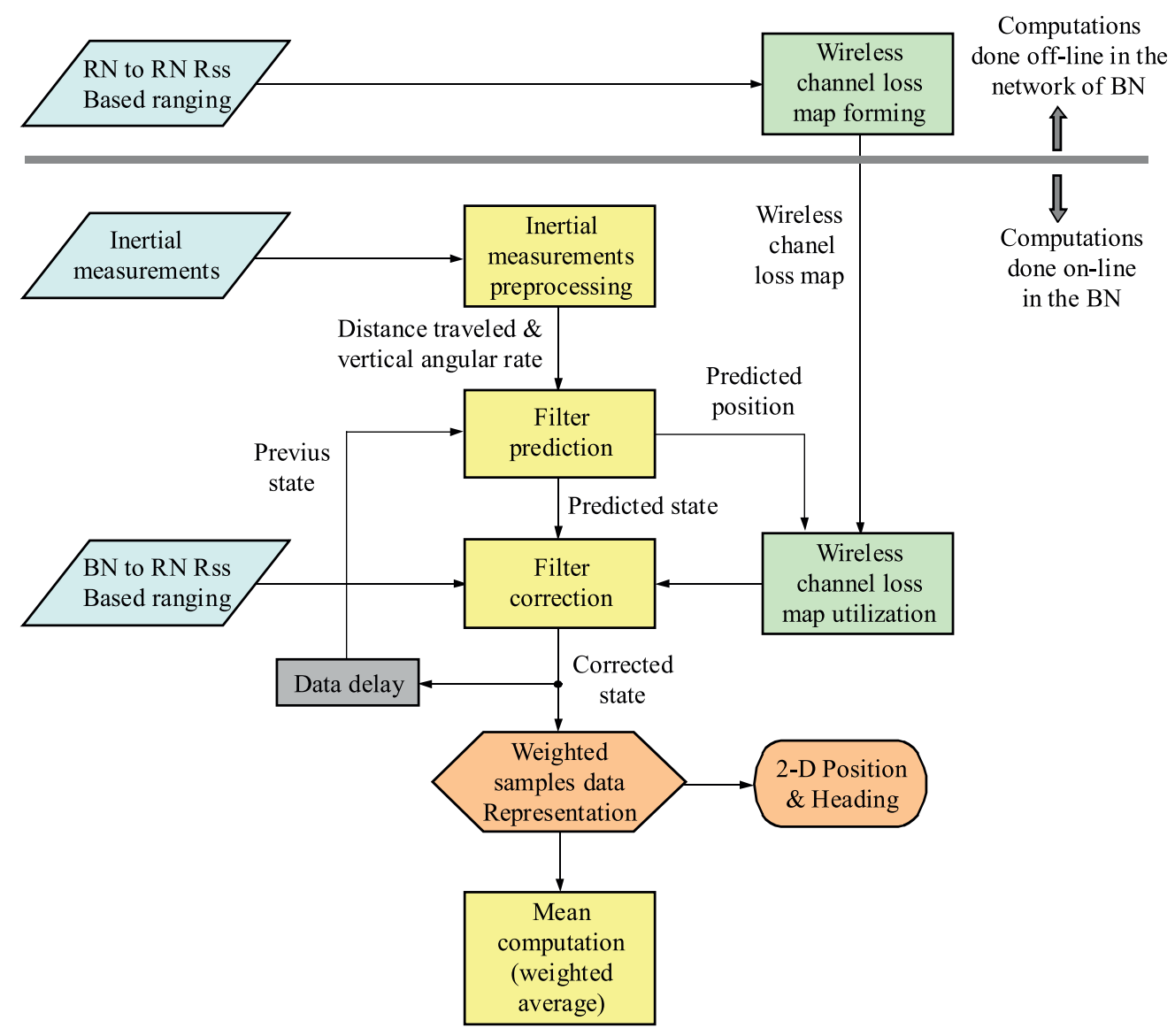

Fig. 5. Complete algorithm flow chart

(multi) loss hypothesis standard deviations are updated accordingly

$$
\begin{aligned}
\hat{\sigma}_{P w, L}^{2} & =\left(\sigma_{P w, L}+N_{a v r} L_{a v r}\right)^{2} \\
\hat{\sigma}_{P w, n L}^{2} & =\left(\sigma_{P w, n L}+N_{a v r} L_{a v r}\right)^{2} .
\end{aligned}
$$

Thought it might be considered also to update the power loss (increase it by $N_{a v r} L_{a v r}$ ) it will not be proposed in order to lower the dual approximation error. This error is due to unmodeled correlations between the $\mathrm{RN}$ to $\mathrm{RN}$ hypotheses and causes the single power loss to be higher.

An example is used to compare the dual (approximative) wireless channel loss mapping and the full (exhaustive) one. For dual wireless channel loss mapping, the belief is represented by a sum of two weighted Gaussian distributions (Fig. 4).

\subsection{Particle Filter Based Pedestrian Navigation Algorithm with Dual Wireless Channel Loss Mapping}

The flow chart of the complete proposed algorithm (Particle Filter Based Pedestrian Navigation Algorithm with Dual Wireless Channel Loss Mapping) can be seen on Fig. 5. Firstly, the wireless channel loss map is off-line formed based on the RSS ranging between all RNs. The map needs to be processed and stored in one central point of RNs.
All other computations are done in the BN. The inertial measurements are preprocessed and the pedestrian (BN) state (position, heading, etc) is predicted based on the previous state, system dynamic model, and the control signals (inertial preprocessing block outputs). The predicted position and dual wireless channel loss map are used to compute the estimate of shadowing power loss for each BN to RN measurement. This shadowing power loss estimate, current RSS measurements, and measurement model are employed to correct the filter prediction. Since the a posterior belief in a PF is represented by a set of weighted samples the means of the estimated states need to be computed finally.

\section{EXPERIMENTAL EVALUATION}

\subsection{Evaluation Tools}

The Matlab simulation environment was selected to evaluate the proposed algorithms. To simulate the realistic sensor readings, two validation tools were employed: Reference measurement data set and I-Prop software.

A reference measurement data set for multisensor pedestrian navigation with accurate ground truth represents a measurement data set for testing and evaluating multi-sensor approaches in pedestrian navigation. The measurements include both transitions from outdoor to 


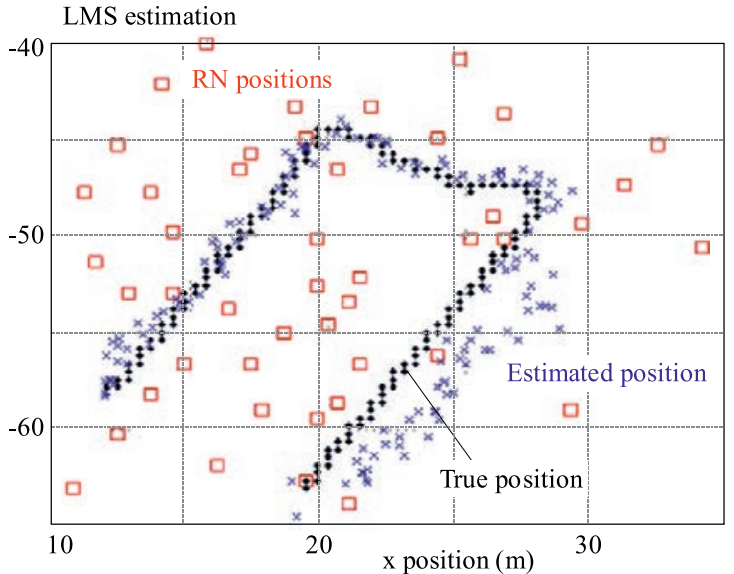

Fig. 6. LMS estimation error

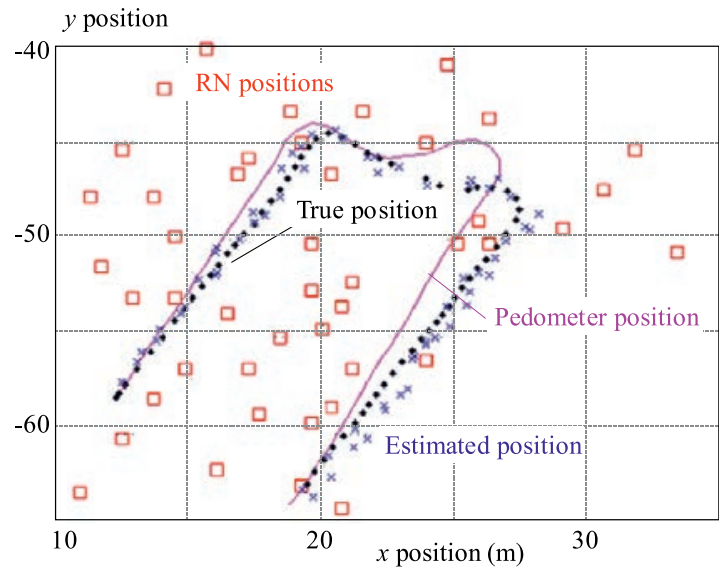

Fig. 8. Eight-state particle filter estimation error

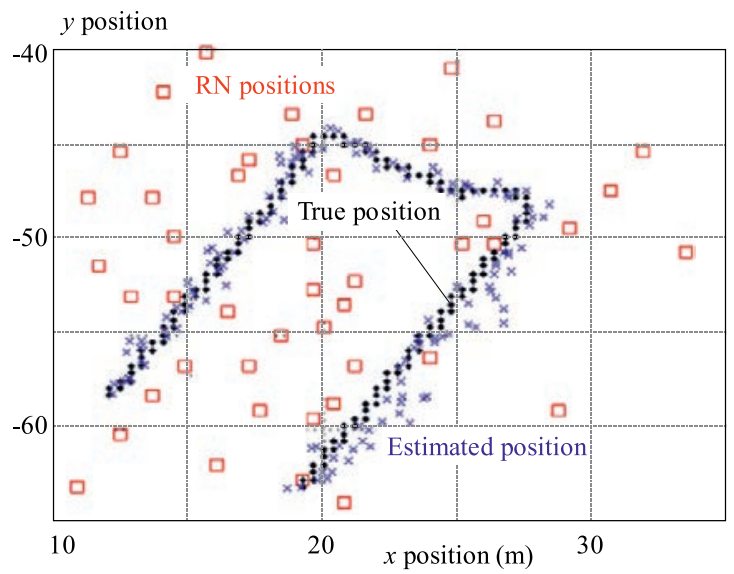

Fig. 7. WLMS \& Dual mapping estimation error

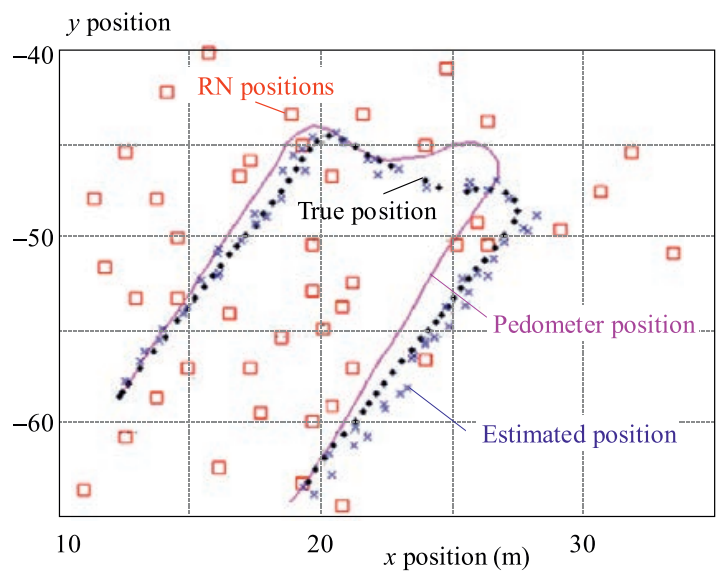

Fig. 9. Eight-state particle filter \& dual mapping estimation error

Table 1. Result of simulations

\begin{tabular}{|c|c|c|c|c|}
\hline Estimation algorithm & LMS & $\begin{array}{l}\text { WLMS \& Dual } \\
\text { mapping }\end{array}$ & 4-state PF & $\begin{array}{l}\text { 4-state PF \& Dual } \\
\text { mapping }\end{array}$ \\
\hline Linear error (m) & 1.063 & 0.601 & 0.717 & 0.660 \\
\hline Root mean square error $(\mathrm{m})$ & 1.291 & 0.726 & 0.832 & 0.745 \\
\hline Estimation algorithm & 6-state PF & $\begin{array}{c}\text { 6-state PF \& Dual } \\
\text { mapping }\end{array}$ & 8-state PF & $\begin{array}{c}\text { 8-state PF \& Dual } \\
\text { mapping }\end{array}$ \\
\hline Linear error $(\mathrm{m})$ & 0.572 & 0.485 & 0.527 & 0.479 \\
\hline Root mean square error $(\mathrm{m})$ & 0.653 & 0.581 & 0.599 & 0.569 \\
\hline
\end{tabular}

indoor and vice versa. The measurements have been carried out in and around a lab and office building. Ground truth reference points are provided with sub-centimeter accuracy [18].

This reference measurement data set is freely provided by the German Aerospace Center (Deutschen Zentrums fuer Luft- und Raumfahrt - DLR) and consists of these sensor measurements: three IMUs (two mounted to the pedestrian foots and one in the pocket of the pedestrian), magnetometer, barometric altimeter, GPS and active RFID tags. Only a part of recorded data will be used in the evaluation, as the pedestrian walks through one floor of an office.
I-Prop is a software tool for coverage planning of indoor wireless systems. Propagation prediction models for multi-floor buildings enable a coverage analysis and design of 3D picocellular networks.

Although the reference measurement data set includes the RFID readings, the signal coverage is very weak and the RSS measurements between the RNs are missing. Since these measurements are crucial for the dual wireless channel loss mapping, the I-Prop environment was combined with the reference measurement data set to form a new data set of logged/ simulated measurements needed for proposed algorithm. 


\subsection{Evaluation Definition}

To evaluate the proposed solution and appropriateness of each design decisions 6 different versions of fused navigation algorithms (PF) are evaluated. The LMS snapshot solution based only on RSS measurements is used as a baseline algorithm and finally, WLMS snap-shot solution based on the RSS measurements and the dual wireless channel loss mapping is used to assess the contribution of the mapping.

The snapshot algorithms were evaluated in every cell of the reference trajectory, but the filtered algorithms were evaluated every time the step was detected. Less evaluation points for filtered algorithms, then for snapshot ones, will be seen, because the step length is typically bigger than the selected grid distance.

\section{RESULTS}

As can be seen in Figures 6-9 and mainly in Table 1, all design decisions were correct; the 8-state PF with dual wireless channel loss mapping is the most accurate estimator and compared to the baseline LMS the accuracy was increased more than twice. It is also interesting that the dual wireless channel loss mapping deployment has comparable performance increase as the fusion with inertial sensors; compare the WLMS with dual wireless channel loss mapping and all the PF without dual wireless channel loss mapping.

One might be surprised by the high estimation accuracy (around 1 or even $0.5 \mathrm{~m}$ ), it is due to high density of simulated RNs. If a smart building, where the control is realized via wireless network, would be assumed the results are representative.

\section{CONCLUSIONS}

In this work, the focus was put on pedestrian navigation systems for indoor and urban areas. The literature survey was conducted first. Appropriate sensor and environment models were selected and their outputs were fused in effective and novel way. The evaluation of proposed solution was done via simulations (Matlab) with usage of external tools (Reference measurement data set for multisensor pedestrian navigation with accurate ground truth and I-prop modeling environment), which assures that not only the fusion algorithms but also the models selected in the solution are assessed independently.

There was developed a navigation algorithm, which is suitable for low-cost pedestrian navigation system for indoor and urban environments where a network of wireless nodes was already installed. The "already installed" should be understood in way that no special RF beacons are required since the algorithm is based on RSS ranging and RSSI is typically available in most modern wireless networks.

$\mathrm{PF}$ was deployed to fuse all the available information. This type of $\mathrm{BF}$ is able to process high non-linearities, which were mainly in the proposed measurement model. The deployment of tightly integrated filter has ensured a high fidelity of the RSS measurement error spatial distribution. A novel and accurate system dynamics model was proposed, too. This model was incorporating correct statistical characteristics (no magic, artificial constants were used), had refined the step length estimation and estimation of the main RF channel parameters.

The RN to RN measurements and their known positions were used to build a wireless channel loss map distributed among the navigation area. The deployment of such a map in navigation algorithms significantly increases the positioning accuracy. There were proposed two versions of wireless channel loss mapping: full (exhaustive) and dual (approximative). The full mapping correctly enumerates the probabilities (weights) of each hypothesis. The complexity of thTe full map forming algorithm is a problem because the number of variables (representing the hypothesis probabilities) can increase up to hundreds in the real applications. Since these variables are heavy correlated a solution of the system of equations needs to be computed; which can be very computationally demanding and so simplified (dual mapping) method was proposed. The discussed computational complexity was also the reason why only the dual (approximative) wireless channel loss mapping was evaluated via simulations and stated in this work. The full/exhaustive mapping was described in [19]. The dual approach approximates all the possible hypothesis by just two (loss/no-loss).

The combination of main RF channel parameters estimation and wireless channel loss mapping represents a novel and very effective way of RSS ranging errors elimination and easily balances out the disadvantage that the proposed solution was not tuned on the real RF data. Such a tuning would also be valid just for the local area where the measurements whould have been collected. On the contrary, the proposed approach enables an automated tuning to arbitrary environments.

Let us switch from qualitative to quantitative conclusions, the proposed algorithm was able to increase the accuracy more than twice as compared to the baseline LMS operating on the RSS measurements! LMS, WLMS, and 6 version of $\mathrm{PF}$ were evaluated. The eight-state PF with dual mapping should be considered as an algorithm proposed in this thesis. Based on the results, it might be considered not to estimate the gyroscope bias. Because the pedometer parameters estimation converged for eight-state PF but the wireless channel parameters only for six-state PF there could be also developed a scheme where the pedometer parameters estimation would be controlled.

Since the evaluation of proposed solution was done via simulations (Matlab) with usage of external tools, it was assured that not only the fusion algorithms but also the models selected in the solution were assessed independently. 
Although only 2-D position estimation was proposed, the difference between transmitter and receiver was compensated in the range measurements. The 2-D approach is well aligned with the wireless channel loss mapping, which is also done in 2-D because the walls (the main sources of channel loss) are typically vertical and are built from the ground to the ceiling. For multi-floor buildings, the navigation algorithm should be supplement with floor detection.

The PF measurement update is triggered by the step detection and a part from the vertical angular rate integration all the states dynamics are distretized with the sampling rate given by this step detection. This fact might be a problem when the pedestrian stops and the integration interval is too long for Euler integration. So for real applications, the system dynamics update triggering should be separated from the measurement update.

\section{REFERENCES}

[1] FLUERASU, A.-JARDAK, N.-VERVISCH-PICOIS, A.SAMAMA, N. : Gnss Repeater based Approach for Indoor Positioning: Current Status, in European Navigation Conference, Global Navigation Satellite Systems, 2009.

[2] KEUNHO, Y.-DAIJIN, K.: Robust Location Tracking using a Dual Layer Particle Filter, Pervasive and Mobile Computing 3 (03 2007), 209-232.

[3] SAVARESE, C.-RABAEY, J. M.-BEUTEL, J. : Location in Distributed ad-hoc Wireless Sensor Networks, in Proc. IEEE ICASSP '01, vol. 4, 2001, pp. 2037-2040.

[4] PATWARI, N.-HERO, A. O.-PERKINS, M.-CORREAL, N. S.-O'DEA, R. J. : Relative Location Estimation in Wireless Sensor Networks, IEEE Transactions on Signal Processing 51 (08 2003), 2137-2148.

[5] PATWARI, N.-ASH, J. N.-KYPEROUNTAS, S.-MOSES, A. O. H. III, R. L.-CORREAL, N. S.: Locating the Nodes [Cooperative Localization in Wireless Sensor Networks, IEEE Signal Processing Magazine 22, (04 2005), 54-69.

[6] FOX, D.-HIGHTOWER, J.-KAUZ, H.-LIAO, L.-PATTERSON, D. J. : Bayesian Techniques for Location Estimation, in Proceedings of the 2003 Workshop on Location-Aware Computing, Oct 2003, pp. 16-18.

[7] GROVES, P. D. : Principles of GNSS, Inertial, and Multi-Sensor Integrated Navigation Systems, Artech House, 2008.

[8] FARRELL, J. : Aided Navigation: GPS with High Rate Sensors, The MacGravy Hill Companies, 2008.

[9] FOXLIN, E.: Pedestrian Tracking with Shoe-Mounted Inertial Sensors, IEEE Computer Graphics and Applications 25 (2005), 38-46.

[10] KRACH, B.-ROBERTSON, P.: Cascaded Estimation Architecture for Integration of Foot-Mounted Inertial Sensors, in IEEE/ION Position Location and Navigation Symposium, 2008, pp. $112-119$.

[11] KUOROGI, M.-KURATA, T.: Personal Positioning based on Walking Locomotion Analysis with Self-Contained Sensors and a Wearable Camera, in Proceedings of the 2nd IEEE/ACM International Symposium on Mixed and Augmented Reality, 2003, pp. 103-112.

[12] ROBERTSOn, P.-KRACH, B.-KHIDER, M.: Slam Dance: Inertial-based Joint Mapping and Positioning for Pedestrian Navigation, Inside GNSS 5 (2010), 48-59.

[13] MATTHEWS, C. J.-KETEMAA, Y.-GEBRE-EGZIABHER, D.-SCHWARTZ, M.: In-Situ Step Size Estimation using a Kinetic Model of Human Gait, in ION GNSS (2010).

[14] Thrun, S.-BURGard, W.-FOX, D.: Probabilistic Robotics, The MIT Press, 55 Hayward street, Cambridge, 2005.

15] COUlson, A. J.-Williamson, A. G.-VAughan, R. G. : A Statistical Basis for Lognormal Shadowing Effects in Multipath Fading Channels, IEEE Transactions on Communications 46 (04 1998), 494-502.

[16] HASHEMI, H.: The Indoor Radio Propagation Channel, Proceedings of the IEEE 81 (1993), 943-968.

[17] SEYBOLD, J. S. : Introduction to RF Propagation, John Wiley \& Sons, Inc., Hoboken, New Jersey, 2005.

[18] ANGERMANN, M.-FRIESE, A.-KHIDER, M.-KRACH, B.-KRACK, K.-ROBERTSON, P.: A Reference Measurement Data Set for Multisensor Pedestrian Navigation with Accurate Ground Truthinbook in European Navigation Conference, Global Navigation Satellite Systems.

19] KAŇA, Z.: Personal Navigation Based on Wireless Networks and Inertial Sensors, Brno University of Technology, Faculty of Electrical Engineering and Communication, 2014, thesis.

Received 17 December 2013

Zdeněk Kaňa received his master degree in Control and Instrumentation from The Faculty of Electrical Engineering and Communication, Brno University of Technology (BUT) in 2006. He is pursuing his $\mathrm{PhD}$ at the same university. His research focus is in multi-sensor navigation with special emphasis in personal localization in indoor/urban areas. He has joined Honeywell International in the position of Scientist in 2010 where he deals with research and development of GNSS, inertial and other sensors based algorithms for aerial navigation and data fusion, including integrity monitoring.

Zdeněk Bradáč is Associated Professor at Department of Control and Instrumentation, Faculty of Electrical Engineering and Communication, Brno University of Technology (BUT) since 2008. He is focused to automation technology, measurement, communication, embedded systems etc. He was involved in more then 51 national and international projects and is a Senior researcher at Central European Institute of Technology (CEITEC).

Petr Fiedler is Associated Professor at Department of Control and Instrumentation, Faculty of Electrical Engineering and Communication, Brno University of Technology (BUT) since 2010. He is focused to control technology, sensors, wireless and wired communication, real-time systems etc. He was involved in more than 20 national and international projects and is a Senior researcher at Central European Institute of Technology (CEITEC). 